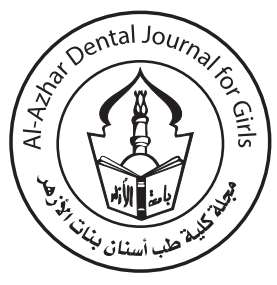

\title{
The Remineralizing Potential of Nano Bioactive Glass Versus Nanohydroxyapatite on Dentine as Affected by PH Cycling*
}

\author{
Dalia M. Elasser ${ }^{(1)}$, Maha A. Niazy ${ }^{(2)}$, Doaa A.E Elsharkawy ${ }^{(3)}$ and Mai Sh. Mansour ${ }^{(4)}$
}

Codex : $38 / 1810$

azhardentj@azhar.edu.eg

http://adjg.journals.ekb.eg

\section{KEYWORDS}

Affected dentin, Bioactive glass, hydroxyapatite, remineralization, fluoride, nanoparticles.

\begin{abstract}
Objective: The aim of this study was to evaluate the remineralizing potential of nano bioactive glass (BG) in comparison with nano hydroxy appetite (HA) incorporated into adhesive on demineralized dentine as affected by $\mathrm{PH}$ cycling. Materials and Methods: sixty sound premolars were extracted for orthodontic treatment. Class I Cavities were prepared in premolars with a depth $3 \mathrm{~mm}$. The specimens were divided into three treatment groups including $20 \%$ BG incorporated into self-etch adhesive (3M ESPE), 20\% HA incorporated also into self-etch adhesive, and gel of sodium fluoride $\mathrm{NaF}$ was used as a positive control. $37 \%$ phosphoric acid was applied for each cavity for lesion creation. Modified adhesive and composite restoration were applied according to the manufacture instructions. An in vitro $\mathrm{pH}$ cycling model was used to mimic oral cavity condition through treatment solutions, acidic buffer (4 hours ) and neutral buffer(20 hours) for 30 days. The specimens were evaluated for radiodensity using Digital Radiographic (DBSW software) and Environmental Scanning Electron Microscope (ESEM) at different stages of the study (before demineralization, after demineralization and after remineralization). The significance level was set at $\mathrm{p} \leq 0.05$. Results: all groups showed significant reminerlization. Groups with BG nanoparticles and HA nanorods showed a higher increase in the radiodensty of demineralized dentine than $\mathrm{NaF}$ group. BG group showed higher results under neutral condition. Conclusion: BG and HA modified adhesives may be valuable tools to enhance remineralization of affected dentin.
\end{abstract}

\section{INTRODUCTION}

Dental caries is a disease caused by the by-products (organic acids) of bacteria in the biofilm on tooth enamel or dentine; the organic acids produced by bacteria damage dental hard tissues and structures, leading

- Paper extracted from thesis titled "The Remineralizing Potential of Nano Bioactive Glass Versus Nanohydroxyapatite on Dentine as Affected by PH Cycling".

1. Demonstrator, operative dentistry Department, Faculty of Dental Medicine for Girls, Al-Azhar University, Egypt.

2. Professor and Head of operative dentistry Department, Faculty of Dental Medicine for Girls, Al -Azhar University, Egypt.

3. Lecturer of Operative Dentistry Department, Faculty of Dental Medicine for Girls, Al-Azhar University, Egypt.

4. Associate Professor, Oral Medicine Department, Faculty of Dental Medicine for Girls, Al-Azhar University, Egypt. 
to tooth decay or cavities. If lesions continually develop in the enamel or even in the dentine under the enamel, it results in deep caries, with two layers in carious dentine. The outer layer is heavily infected by microorganisms and is broken down to the extent that it cannot be remineralized at all. However, the inner layer, immediately adjacent to sound dentine on the floor of the lesion, will be partially demineralized, will contain some bacterial flora, but will still have some of the original dentine tubule structure present. This was identified as the affected layer ${ }^{(1)}$. Traditional treatment of carious teeth, involving removal of all carious tooth structure prior to placement of the restorative material, often sacrifices more structure than necessary ${ }^{(2)}$. Other authors have shown evidence of carious dentine remineralization, of the same deep remaining layer, by means of different methodologies and utilizing several materials ${ }^{(3)}$.

Different dentin remineralization strategies have been investigated, most of which focus on the use of bioactive glass, fluoride-releasing materials, casein phosphor peptide-amorphous calcium phosphate (CPP-ACP) complexes, and calcium hydroxide. An alternative strategy, that is becoming the focus of much research in this field, is the use of nanoparticles for the management of dental caries. Nano particulate hydroxyapatite, nano-sized carbonated apatite (n-CAP), carbonatehydroxyapatite nano-crystals (CHA) and nanoparticulate bioactive glass are among those nanomaterials that seem to increase the mineral content of enamel and/or dentin. A significant challenge with the use of these materials is to achieve an effective and deep infiltration of the intact demineralized dentin collagen with the particles, while avoiding precipitation on the surface ${ }^{(4)}$.

Bioactive glass (calcium sodium phosphor silicate) acts a biomimetic mineralizer, i.e., it simulates the body's own natural bio mineralization process ${ }^{(5)}$.The basic building block of the enamel is hydroxyapatite. The nano-sized crystals of hy- droxyapatite thus provide a unique window for the restoration of the demineralized enamel. However nowadays, it has been used in remineralizing dentifrices to restoring the structural integrity of initial carious enamel ${ }^{(6)}$. Compared to the typical hydroxyapatite; nano-hydroxyapatite has a greater solubility, greater surface energy, and higher biocompatibility $^{(7)}$. This study aims to investigate the effect of incorporation of nano hydroxyapatite and nano bioglass particles into dental adhesive on remineralization of affected dentin.

\section{MATERIALS AND METHODS}

A total of 60 freshly extracted sound human premolars extracted due to orthodontic treatment from patients with average age 18 to 25 years old were included in this study. Ethical approval for the use of extracted human teeth was obtained in accordance with Guide lines from research ethics committee approval of Dental Medicine Faculty -Al-Azhar University for girls. Teeth with cracks, fractures, carious lesion of the crown or the root, restorations and previous endodontic treatment were excluded. The teeth were stored at $4^{\circ} \mathrm{C}$ in thymol solution until used. They were used not more than 1 month post-extraction ${ }^{(8)}$. The samples were randomly divided into three groups of twenty each according to type of material to be used; the first group is BG nanoparticles incorporated (A1), the second HA nanorods incorporated (A2) and in the third group flouride gel was applied(A3).

Each of the three groups was further subdivided into two subgroups of ten each according to subjection to $\mathrm{PH}$ cycling or not; the first group was subjected to ph cycling (B1), the second was not subjected to ph cycling(B2). Such demarcation was made to allow for assessment at baseline, after demineralization and post treatment.

\section{Preparation of nano particles}

Bioactive glass nanoparticles were synthesized by using alkoxide sol gel technique while Hydroxapatite nano rods were synthesized by wet 
chemical reaction method and preparation of nano grade calcium Phosphate (Ca2PO4). characterization of hydroxyapatite nanorods and bioactive glass nano particles was done by using Transmission Electron Microscope.

\section{Samples Preparation}

Circular cavities were prepared in the center of the occlusal surface of each premolar which were standardized (depth=3 mm) using a small sized carbide round bur mounted in a high speed hand piece under copious water coolant. The size of the cavities was checked with a digital caliber. Tungsten carbide burs \#2 with an initial depth of $1.5 \mathrm{~mm}$ were used to reach the final size set at $3 \mathrm{~mm}$ with a diamond stone \#3 at high rotation, under continuous cooling. After 8 cavities, drills were replaced by new ones ${ }^{(9)}$. Baseline radiodensity was evaluated at base of the cavity. The obtained specimens were stored in deionized water at room temperature $22 \pm 20 \mathrm{c}$ to prevent dehydration of the teeth till the next step.

\section{Preparation of adhesives}

For mixing the single bond universal adhesive with BG nanoparticles, the universal single bond was put into tubes wrapped with dark adhesive tape to protect it from exposure to the light. For the control group, the adhesive was used as supplied by the manufacturer. The amount of each material to be mixed with the bond was calculated according to the following equation (weight volume\%=weight of solute volume of solution $\times 100(10)$.

The weight of BG (20 wt. \%) was 0.2gm.and the weight of HA (20 wt. \%) was also calculated and the result was $0.5 \mathrm{gm}$ for each. An electronic balance (A\&D Company Limited, Japan) was used to measure the amount of each material. Each material was transferred to its specific tube and covered securely to be ready for mixing. This process was done in the developing dark room. Each tube was given a number corresponding to each group.
To achieve complete dissolution of $\mathrm{BG}$ and dispersion of the nanoparticles, the adhesive mixture was mixed for 60 seconds by amalgamator (D-650N digital amalgamator) ${ }^{(5)}$. For complete dissolution of $\mathrm{HA}$, the adhesive mixture was mixed for 15 minutes by ultrasonicator (100B-HB ultrasonic processor, USA )

\section{Application of the adhesive system and composite restoration}

Phosphoric acid gel (Fine Etch37) 37\% was applied on the walls of the prepared circular cavity for 2 minutes for dentin deminealization. Radiodensity was evaluated. Two groups (Groups A1 and A2) were treated by using modified universal bond (self etch technique),which were filled with the nanoparticles. While in group A3, NaF gel was applied then removed from the dentin surface, then the cavity was restored using unmodified adhesive. The steps were the same of application for the three groups. The adhesives were applied according to the manufacture instructions. All the specimens were restored with Filtek TM 350 XT composite according to the manufacture instructions.

\section{pH cycling}

Each group was subdivided into two sub groups according to $\mathrm{pH}$ cycling. One of them was subjected to $\mathrm{PH}$-cycling and other subgroup was immersed in artificial saliva. For $\mathrm{pH}$ cycling, the specimens were exposed to a daily cyclic treatment regime which involved exposing the specimen to de- and remineralizing solutions. The remineralizing solution was natural solution (physiological solution of Ringer ) and the $\mathrm{pH}$ was adjusted to7. Demineralizing solution contained $1.5 \mathrm{mmol} / \mathrm{L} \mathrm{CaCl} 2,0.9 \mathrm{mmol} / \mathrm{L} \mathrm{KH} 2 \mathrm{PO} 4,50$ $\mathrm{mmol} / \mathrm{L}$ lactic buffer, with $\mathrm{pH}$ 5. The $\mathrm{PH}$ of the solutions was measured using $\mathrm{PH}$ meter (PH 315i/ SET) Specimens were placed in an incubator at $37^{\circ} \mathrm{C}$. Each specimen was immersed in $10 \mathrm{ml}$ of remineralising solution for 20 hours at $37^{\circ} \mathrm{C}$, removed and washed with de-ionized water and then 
immersed in $10 \mathrm{ml}$ of demineralizing solution within another vial for 4 hours at 37 . The cycling program was carried out for 28 days ${ }^{(11)}$. The demineralizing and remineralizing solutions were changed on the fourth day of $\mathrm{pH}$ cycling ${ }^{(12)}$. Artificial saliva; A physiological solution of Ringer was prepared in the laboratory from Sodium Chloride, $\mathrm{NaCl}$ 9.0mg, Potassium Chloride( $\mathrm{KCl} 0.4) \mathrm{mg}$, Calcium chloride hexahydrate, $\mathrm{CaCl} 2-6 \mathrm{H} 2 \mathrm{O} 0.2$ and Sodium hydrogen carbonate, NaHCO3 0 and $1000 \mathrm{ml}$ of deionized water. $10 \mathrm{~N}$ sodium hydroxide was added to this mixture until the $\mathrm{pH}$ value was measured to be as $6.75 \pm 0.15^{(13)}$. The solution was shook well every day and changed every.

\section{Assesment of Remineralization}

The radiodensity of each sample was measured using digital radiography. Standardized reproducible periapical radiographs for each sample were done after cavity preparation, after demineralization and one month after restoration. The DBS-win software, which is a part of the Vista Scan system was followed in this study to obtain and calculate pixel gray measurement (mineral content change) using the image processing software. An x-ray machine with $70 \mathrm{kV}$ and $0.5 \mathrm{~mA}$ dose for 0.04 seconds was utilized in imaging. The exposed image plate was used to transmit the images of the samples to the digital scanner and accordingly, to the computer. The saved images of each sample were interpreted to evaluate and record the pixel gray measurement (mineral content change) by two examiners. In each sample a line was drawn along the CEJ to act as a reference, while another parallel line was drawn at the bottom of the cavity. The distance between both lines was standardized (for each sample) by measuring the length of a vertical line connecting them to be reproducible. Radiodensity along the line representing bottom of the cavity was measured at three points (at its start, middle and end).The average radiodensity was identified by taking the corresponding three readings from the DBSWin software and calculating the mean.
These procedures were done at baseline then repeated after demineralization then after 1month after reminerlization. The mineral density mean value was calculated from the equation (average density $=$ average intensity/ profile); (the profile was determined directly in the utilized software).

\section{Scanning Electron Microscope:}

Representative samples from each group were chosen to examine the micro-morphological changes that occurred in the dentin after etching and after the application of the remineralizing solutions. The specimens were prepared by sectioning each specimen mesiodistally into buccal and lingual divisions for interface examination (between adhesive layer and dentin surface). The sections were then demineralized for 30 seconds with $6 \mathrm{~N} \mathrm{HCl}$, rinsed again, deproteinized with $2.5 \%$ $\mathrm{NaOCl}$ for 10 minutes, and then serially dehydrated with $25,50,75,95$ and $100 \%$ ethanol for $15 \mathrm{~min}$ each ${ }^{(71)}$. Next, the specimens were mounted on aluminum stubs, then examined under SEM (Field Emission Gun), with accelerating voltage 30 K.V., magnification $14 x$ up to 1000000 and resolution for Gun.1n.

\section{Statistical Analysis}

The data was obtained, recorded, tabulated and statistically analyzed. The data were analyzed by computer using statistical program SPSS ver.17.0* (Statistical Package for Scientific Studies). Numerical data were presented as mean and standard deviation values. Mean and standard deviation values were estimated of quantitative data. Data analysis was performed using one way ANOVA, pair-wise Tukey's post-hoc and Student t-tests.

\section{RESULTS}

Comparing the changes of mean values with different materials in $\mathrm{pH}$ cycling (BG, $\mathrm{HA}, \mathrm{NaF})$ a higher mean value was recorded in remineralization within HA groupA2(95.4\%), followed by BG group 
was recorded (73.96\%), while $\mathrm{NaF}$ group recorded the lowest mean(59.41\%). ANOVA test revealed that the difference between groups was statistically significant $(p=0.0056)$ but Bioactive glass group was not significantly different from $\mathrm{NaF}$ group.

Comparing the changes of mean values with different materials in artificial saliva (BG, $\mathrm{HA}, \mathrm{NaF}$ ) the highest mean value was recorded in $\mathrm{BG}$ group (115\%), followed by HA group ( $81.3 \%$ ), while the least mean value was recorded in $\mathrm{NaF}$ group (32\%). ANOVA test revealed that the difference between groups was statistically significant $(\mathrm{p}<0.0001)$. Table (1) Fig(1)

\begin{tabular}{|c|c|c|c|c|c|}
\hline & & Group & Group & Group & \\
\hline $\mathrm{pH}$ & Change in & $73.96 \% \pm$ & $95.4 \% \pm$ & $59.41 \% \pm$ & $0.0056 * *$ \\
cycling & mean (\%) & 16.582 & 17.681 & 14.264 & \\
\hline Artificial & Change in & $115.13 \% \pm$ & $81.03 \% \pm$ & $32.39 \% \pm$ & $0.0001 * * *$ \\
saliva & mean(\%) & 19.206 & 21.011 & 13.205 & \\
\hline
\end{tabular}

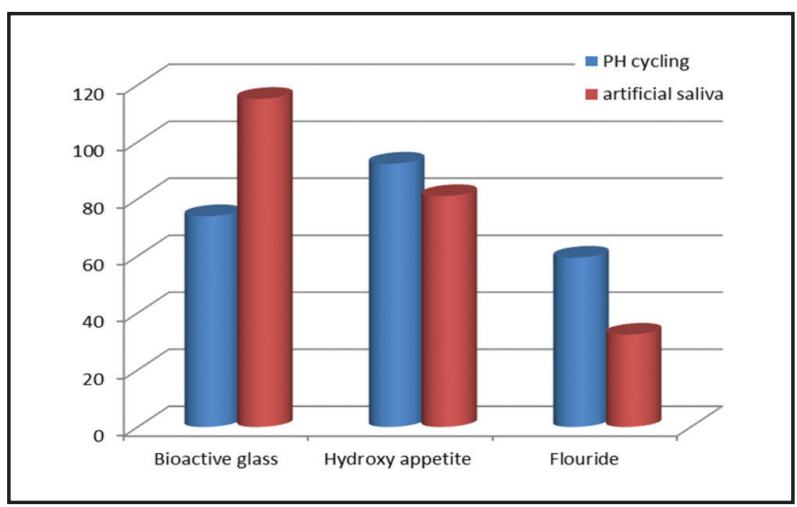

Fig. (1): Column chart represent percent change of mean values and SD for demineralization and remineralization of dentin radiodensity illustrate the effect of $\mathrm{PH}$ cycling on dentin reminerlization with different materials.

The observation of the photomicrographs resulting from backscattered electron mode at magnification of $2000 \mathrm{X}$ revealed that there was mineral precipitation in all the specimens that had different patterns Figures (1:4) show representative ESEM photomicrographs which have been interpreted as follows:

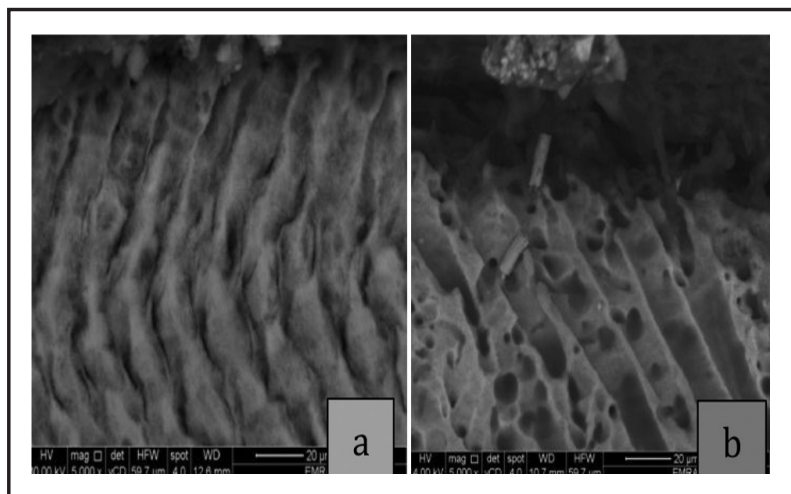

Fig. (2) showing: a) normal dentin, b) deminerlized dentin treated with unmodified adhesive

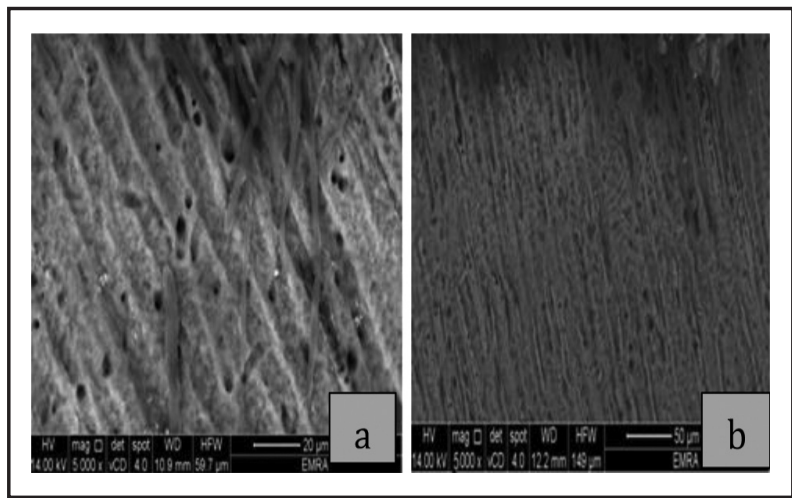

Fig. (3) Showing: a, demineralized dentin treated with BG then stored in $\mathrm{pH}$ cycling for 1 month, b,demineralized dentin treated with $\mathrm{BG}$ then stored in artificial saliva for 1 month

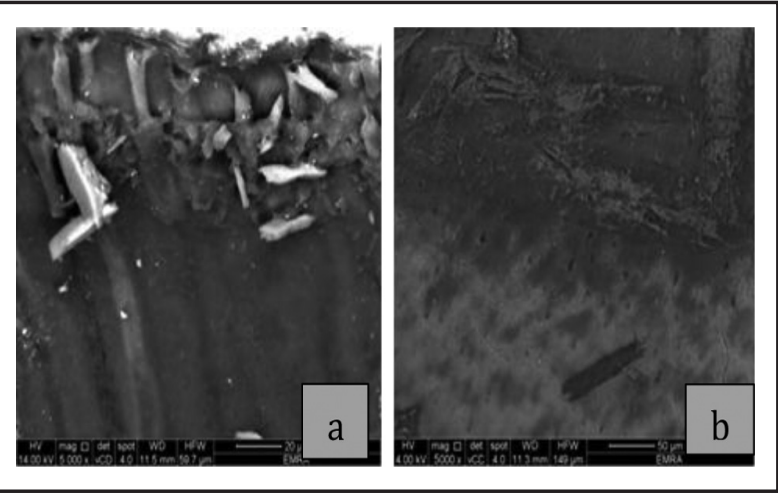

Fig. (4) Showing: a, demineralized dentin treated with HA then stored in $\mathrm{pH}$ cycling for $1 \mathrm{month}, \mathrm{b}$, demineralized dentin treated with HA then stored in artificial saliva for 1 month 


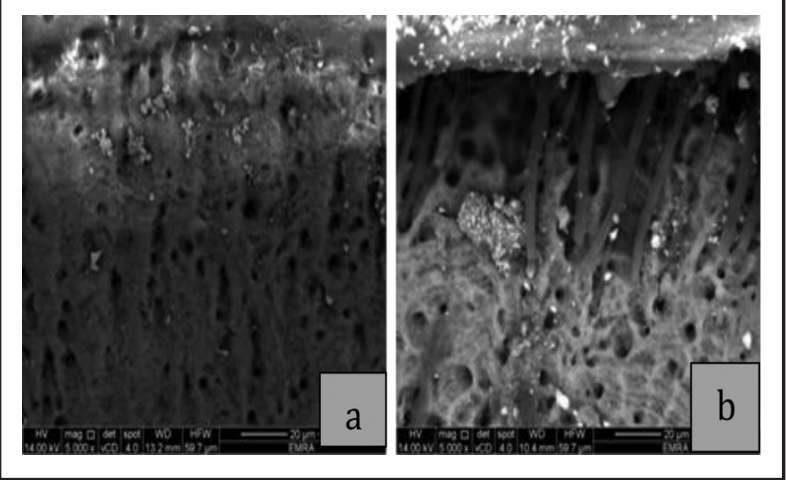

Fig. (5) showing:a, demineralized dentin treated with $\mathrm{NaF}$ then stored in $\mathrm{pH}$ cycling for 1month, $\mathrm{b}$, demineralized dentin treated withNaF then stored in artificial saliva for 1 month

\section{DISCUSSION}

Process of dental caries involves demineralization of dentin and bacterial invasion. Dentin is altered on account of acids produced by bacteria, both in its inorganic and organic constituents. These acids lead to more dentin tissue demineralization, with subsequent loss of mineral crystals ${ }^{(14)}$. The modern treatment of carious lesions involves the removal of only the infected external dentin, while internal affected dentin is preserved. Therefore, the objective of the present study was to evaluate the remineralization potential of BG nanoparticles versus HA nanorods incorporated into adhesive on demineralized dentin. The effect of PH cycling was also investigated. Bioactive glass (BG) is a highly biocompatible calcium sodium phosphosilicate, This unique material has numerous novel features, most important of which are its ability to act as a biomimetic remineralizer, due to the matching ability to the body's own mineralizing trial, Hydroxyapatite HA nano rods was used in this study. It is considered one of the most biocompatible and bioactive materials. It is widely applied in demonstrated that nanosized particles have similar morphology and crystal structure compared with dental apatite ${ }^{(17)}$. Concentration of $20 \%$ bioactive glass nanoparticles into the adhesive system was selected in this study as this concentration was shown in the previous studies to cause $\mathrm{Ca} / \mathrm{P}$ precipitations on dentin specimens ${ }^{(18)}$. While concentration of $20 \%$ hydroxyapatite nano rods into the adhesive system was chosen as this concentration was shown in previous studies to improve dentin acid resistance ${ }^{(19)}$. Also $20 \%$ was the same concentration used for BG for elimination an extra variable. The present study used a $\mathrm{pH}$ cycling model, for creation of a condition similar to the oral cavity. The acidic and neutral values were chosen because of the wide $\mathrm{pH}$ range found in the oral cavity due to the consumption of different types of food and liquids associated with different life styles and eating habits ${ }^{(20)}$.

When comparing the materials under different $\mathrm{PH}$ cycling condition, results showed that there was a statistical significant increase in the percent change of dentin radio-density value (post treatment) in HA nanorods group when exposed to $\mathrm{PH}$ cycling than BG nanoparticles group. This is in agreement with another study ${ }^{(20)}$ which found that surface micro hardness increased significantly with decreasing $\mathrm{pH}$ below 7.0 and the most efficient group was at $\mathrm{pH}$ 4.0. When compared with neutral group. This was an indication for more minerals deposited in the inner portion of the lesion when the $\mathrm{pH}$ value was reduced from 7.0 to 4.0. These results in the $\mathrm{pH}$ study may also be directly related to the solubility of nano HA increased in the acidic condition this also supported by another study ${ }^{(21)}$. Numerous studies have demonstrated that an acidic solution has a greater capacity to enhance the penetration of mineral ions during remineralization compared with a neutral solution. However, when comparing BG nanoparticles group with HA nanorods under neutral conditions, results showed that there was a statistical significant increase in the percent change of dentin radio-density value (post treatment) in BG nanoparticles group when exposed to AS than HA nanorods group. This was supported by another study ${ }^{(22)}$ which reported that the highly alkaline conditions play a role in the dissolution and remineralization kinetics. This may be due to high $\mathrm{pH}$ which allows a higher silica concentration in solution. 
Furthermore, the highly deprotonated silica species at high $\mathrm{pH}$ induce greater $\mathrm{Ca} / \mathrm{P}$ precipitation than their counterparts at neutral $\mathrm{pH}$. These results also matched SEM observation where BG nanoparticles treated group showed complete occlusion of the opened dentinal tubules, there was also newly formed precipitates inside the dentinal tubules and a very little dentinal tubules were still opened when the specimens were exposed to AS, but when exposed to $\mathrm{pH}$ conditions, the dentinal tubules were partially occluded and newly formed precipitates inside the dentinal tubules were also found. In HA nanorods treated group, showed all of the dentinal tubules were occluded completely and newly formed precipitates inside the dentinal tubules was also observed when exposed to $\mathrm{pH}$ cycling, but when exposed to AS condition almost of dentinal tubules were occluded with newly formed precipitates inside dentinal tubules.

\section{CONCLUSIONS}

Under the conditions of the present study, the following can be concluded:

1. Modification of adhesives with nanoparticles could be a valuable tool for dentine reminerlization.

2. HA nanorods and $\mathrm{NaF}$ have higher efficacy in more acidic condition, while BG nanoparticles was most effective under neutral condition.

3. PH conditions in the oral cavity play a role in efficiency of different material, in dentine reminerlization.

\section{RECOMMENDATIONS}

1. Further in vivo studies should be done to evaluate the effect of bioactive glass on tooth structure for enamel and dentine

2. The insertion of BG nanoparticles and HA nanorods in adhesive systems as an alternative to preserve the longevity of the restoration, by caries prevention although having promising results, requires further reviews, due to the possibility of changing adhesive durability and bond strength.

\section{REFERENCES}

1. Ngo HC, Mount G, Mc Intyre J, Tuisuva J, Von Doussa RJ. Chemical exchange between glass-ionomer restorations and residual carious dentine in permanent molars: an in vivo study. J dent. 2004;34608-13.

2. Yoshiyama M, Doi J, Nishitani Y, Itota T, Tay F, Carvalho R, Pashley D. Bonding ability of adhesive resins to caries affected and caries infected dentin. J Appl oral Sci 2004: 12:171- 6 .

3. Conrado C A. Remineralization of carious dentine: In vitro microradiographic study in human teeth capped by calcium hydroxide. Braz Dent J 2004: 15: 59- 62.

4. Besinis A, van Noort $R$, MartinN. Infiltration of demineralized dentin with silica and hydroxyapatite nanoparticles. Dent Mater.2012;28:1012-23.

5. Prabhakar AR, Arali V. Fluoride and bioactive glass using bioerodible gel systems. J Dent Res Dent Clinic Dent Prospects 2009;3:117-21.

6. Mishra P, Palamara JE, Tyas MJ, Burrow MF. Effect of loading and $\mathrm{pH}$ on the subsurface demineralization of dentin beams. Calci Tis Intern. 2006;79:273-7.

7. Yamaguchi $\mathrm{K}$, Miyazaki $\mathrm{M}$, Takamizawa $\mathrm{T}$, Inage $\mathrm{H}$, Moore BK. Effect of CPP-ACP paste on mechanical properties of bovine enamel as determined by an ultrasonic device. J Dent. 2006;34:230-6.

8. Cao CY, Mei ML, Li QL, Lo EC, Chu CH. Methods for biomimetic remineralization of human dentine: a systematic review. Inter J molecul Sci. 2015;16:4615-27.

9. Nunes MF, Swift EJ, Perdigao J. Effects of adhesive composition on microtensile bond strength to human dentin. Amer J Dent. 2001;14:340-3.

10. www.physiologyweb.com/calculators/percent_solutions_ calculator.html.

11. Prabhakar AR, Manojkumar AJ, Basappa N. In vitro remineralization of enamel subsurface lesions and assessment of dentine tubule occlusion from $\mathrm{NaF}$ dentifrices with and without calcium. J Ind Soci Pedo Prev Dent. 2013;31:29-35.

12. Bali P, Prabhakar A R and Basappa N. Invitro comparative evaluation of compressive strength and antibacterial activity of conventional GIC and hydroxyapatite reinforced GIC in different storage media .J Clinic Diag Res 2015; 9:51-55.

13. Nucã C., Bocskay T., Amariei C. and Rusu L. Study regarding the histological features of enamel caries. Oral H \& Dent Manag Black Sea Countr. 2005; 4: 5-12. 
14. Mehdawi I, Abou Neel EA, Valappil SP,Palmer G, Salih V, Pratten J, Spratt DA, Young AM. Development of remineralizing, antibacterial dental materials. Acta Biomater. 2009; 5: 2525-39.

15. Mahmoud N, Riad M, Farag A. Remineralizing potential of hydroxyapatite and fluoride nanoparticles on dentin. Egypt dent J. 2015;61:1872.

16. Featherstone JD, Doméjean S. The role of remineralizing and anticaries agents in caries management. Adv Dent Res 2012;24:28-31.

17. Hannig M, Hannig C. Nanotechnology and its role in caries therapy. Adv Dent Res 2012;24:53-7.

18. Khvostenko D, Hilton TJ, Ferracane JL, Mitchell JC, Kruzic JJ. Bioactive glass fillers reduce bacterial penetration into marginal gaps for composite restorations. Dent Mater. 2016;32:73-81.

19. Haghgoo R, Ahmadvand M, Moshaverinia S. Remineralizing Effect of Topical NovaMin and Nano-hydroxyapatite on caries-like Lesions in Primary teeth. J Dent Pract. 2016;17:645-9.

20. Pereira J, Martineli A,Tung M. Replica of human dentin treated with different desensitizing agents. A methodological SEM study in vitro. Braz Dent J.2002;13(2):75-85. 21 Tschoppe P, Zandim DL, Martus P, Kielbassa AM.Enamel and dentine remineralization by nano-hydroxyapatite toothpastes. J Dent.2011; 430-7.

21. Wang Z, Jiang T, Sauro S, Wang Y, Thompson I, Watson TF, Sa Y, Xing W, Shen Y, Haapasalo M. Dentine remineralization induced by two bioactive glasses developed for air abrasionpurposes.JDent.2011; 39:746-5 